\title{
Determination of Lubricant Layer Thickness with an Allowance for Oil Absorption Capacity of Contacting Surfaces
}

\author{
Dmitry Terentyev ${ }^{1}$, Sergey Platov ${ }^{1}$, Maxim Kharchenko ${ }^{1, *}$, Nikolay Ogarkov ${ }^{1}$, Roman \\ Dema $^{1}$, and Elena Suvorova ${ }^{1}$ \\ ${ }^{1}$ Nosov Magnitogorsk State Technical University, 455000, Magnitogorsk, Lenin str., 38, Russia
}

\begin{abstract}
The paper describes an analytical model for calculatinganextreme pressure lubricant layer thickness with an allowance for oil absorption capacity of contacting surfaces. The model is practically assessed in laboratory conditions. The experimenting work shows that the speed of the relative displacement of contacting surfaces and the oil absorption capacityof their rough layers contributes to the thickening of the lubricant. The analysis of the results shows that it is the oil absorption capacity of rough layers that has the strongest influence on the change in the thickness of theextreme pressure lubricant layer.
\end{abstract}

\section{Introduction}

The main reason for a failure of units and mechanisms is not abreakdown of parts under the influence of increased or peak loads, but the wear of contacting surfaces. Therefore, anincrease inthelifetime of heavy-duty friction assemblies is one of the priority aspects in design and exploitation of machine units and parts.

Numerous studies show that friction surfaces withoil-retaining geometry have significantly better tribotechnical properties [1-4], and the insufficient oil absorption capacityof contacting surfacescan resultin adhesion and more intensive wear of friction assemblies in the course of breaking-in and wear of microprotrusions.

In most cases, oil absorptioncapacity is understood as an ability of a surface to accumulate a certain amount of lubricant, which goes to a friction contact in the event of oil starvation. As for the work of a friction couple (in the event of a relative displacement of contacting surfaces), oil absorption capacity is the most important and essential property if we speak about real processes of tribology application in metallurgical and othertypes of equipment.

To increase oil absorption capacity of rough surfaces, various ways of forming oil retaining micro relievesare used. The micro relieves look like a system ofgrooves and cavities with a closed or open profile to accumulate a lubricant $[4,5]$.

Thus, oil absorption capacity of rough surfaces plays a positive role in the interaction of friction surfaces of heavy-duty friction assemblies. However, the reviewed researches don't

\footnotetext{
*Corresponding author: kharchenko.mv@bk.ru
} 
provide with complete information on the quantitative data of the influence of oil absorption capacity of contacting surfaces on thelubricant film formationand the life extension of friction assemblies.

That is why theoretical and experimental researches focused on the evaluation of the dependence of an oil absorption surface and its influence on the formation of a lubricant layer between contacting surfaces become especially important.

The aim of the paper is to find the dependence of an oil film thickness on operational modes of heavy-duty friction assemblies regarding oil absorption capacity.

The carried out analytical model forcalculatinga lubricant layer thickness in heavy-duty friction assemblies is based upon the contact hydrodynamic lubrication theory, theory of elasticity, and the task of the mechanics of contact interaction of elastic bodies.

We use the solution of Flamant problem of displacement of an edge of a smooth semiplanewith an equivalent elasticity modulus at a coordinate $\mathrm{x}$, being under pressure with continuous distribution.In this case, the displacement of a point of an edge of a semi-plane can be described with an equation:

$$
\delta(x)=-\frac{4}{\pi E^{\prime}} \int_{S_{1}}^{S_{2}} P(S) \ln (x-S)^{2} d S+C
$$

where $\mathrm{S}$ is an additional coordinate along $\mathrm{x}$ axis, $\mathrm{C}$ is a constant.

The form ofa gap between contacting bodies is defined by displacement of points of a semi-plane edge regarding an arbitrary point of contact.That is why, for a specific value of a contact region, constant $\mathrm{C}$ in the formula can be set to 0 .

According to A.N. Grubin's theory, we suppose that the deformation of a cylinder in case of a dry contact and in case of feedinga lubricant to a contact zone aresimilar (fig.1).

According to Hertz's theory, the value of displacement of points of an elastic bodyoutside a contact zone will be

$$
\delta_{2}=h-h_{0}=\frac{4 W}{\pi E_{2}^{1}}\left[\sqrt{x\left(x^{2}-1\right)}-\ln \left(x-\sqrt{x^{2}-1}\right)\right]
$$

where $\mathrm{W}$ is a load per unit contact surface, forcing the corresponding displacements.

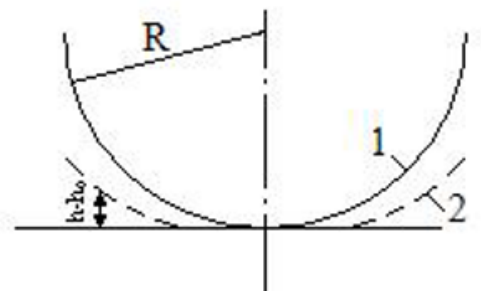

a

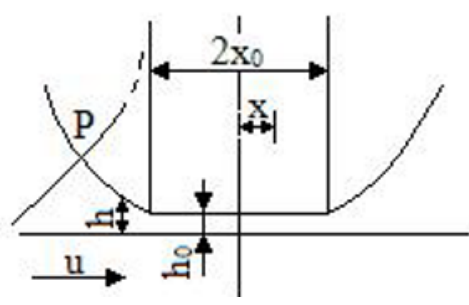

$b$

Fig. 1. The geometry of a surface contact a) without a lubricant b) with a lubricant.

1 and 2 are non-deformed and deformed cylinders correspondingly.

Under high pressure values, which are specific to heavy-duty friction surfaces, there appears a dependence of lubricantviscosity onthe pressure in a contact zone. This dependence has anexponential character for the majority of lubricants.

Upon integrating, Reynolds' equation looks as

$$
\frac{d P}{d x}=12 \eta v \frac{h-h_{0}}{h^{3}}
$$


Inserting the expression of the dependence of viscosity of a lubricant on the pressure $\eta=\eta_{0} e^{\alpha P}$ into the equation (3) and dividing the variables, we get:

$$
\frac{d P}{d x} e^{-\alpha P}=12 \eta_{0} v \frac{h-h_{0}}{h^{3}}=\frac{d q}{d x}
$$

The right part of the equation (4) is a pressure gradient in a film at constant lubricant viscosity. The value $q$ is called reduced pressure. Integrating equation 4 regarding the boundary conditions $\mathrm{P}=0$ at $\mathrm{q}=0$, we get a dependence between the values $\mathrm{P}$ and qin the form

$$
q=\frac{\left(1-e^{-\alpha P}\right)}{\alpha}
$$

The received equation shows that provided $q \rightarrow \frac{1}{\alpha}$, insignificant changes of a film form can result in great changes of pressure. This property demonstrates the validity of the equality of dry and lubricated contacts assumed by A.M. Ertel and A.I. Gubin.

Regarding the assumed correlation $q \approx \frac{1}{\alpha}$, the equation (4)is worked out to

$$
\frac{d q}{d x}=\frac{48 v}{E_{\ni}} \sqrt{\frac{W}{2 \pi E_{\ni} R}} \frac{h-h_{0}}{h^{3}}
$$

where $\mathrm{R}$ is an equivalent of the radius of curvature, defined by the correlation $\frac{1}{R}=\frac{1}{R_{1}}=\frac{1}{R_{2}}$

The assumed pressure at a boundary of a joint:

$$
q_{x=-1}=\frac{48 v}{E_{\ni}} \sqrt{\frac{W}{2 \pi E_{\ni} R}} \int_{\infty}^{-1} \frac{h-h_{0}}{h^{3}} d x
$$

The numerical integration of equation 7 with the use of aquadrature and Gauss multipliers results in:

$$
q_{x=-1}=4,36 \frac{v}{E_{\ni}} \sqrt{\frac{W}{2 \pi E_{\jmath} R}}\left(\frac{\pi}{2 W}\right)^{2}\left(\frac{\pi h_{0}}{2 W}\right)^{-1,375}
$$

The pressure at a boundary of theregion of a static contact $(x=-1)$ should be compared with Hertz maximum pressure, i.e. the assumed pressure reaches the value $\frac{1}{\alpha}$. Replacing the left part of equation 8 by $\frac{1}{\alpha}$ and then solving it regarding the parameter of a lubricant layer thickness at the contact of bodies with rough surfaces, we get

$$
h_{0}=0,888(\alpha \eta v)^{0,727} R^{0,364}\left(\frac{E_{\ni}}{W}\right)^{0,111}
$$

Regarding the oil absorption capacity of a rough layer (2.12) the final thickness of a lubricant film is

$$
h_{0}=0,888\left(1+m^{\prime}+m^{\prime \prime}\right)(\alpha \eta v)^{0,727} R^{0,364}\left(\frac{E_{\ni}}{W}\right)^{0,111}
$$


Taking into account oil absorption of rough layers, this dependence makes it possible to calculate a lubricant layer thickness, when the surfaces are in contact with a relative displacement.

To determine the validity of the mathematical model for calculating a lubricant layerthickness, experimental studies are carried out on laboratory equipment.

The experimental researches of a lubricant layer thicknessbetween contacting surfaces and the evaluation of validity of the developed analytical model

\section{Research materials and methods}

In the course of further experimental studies, a laboratory setup (SMC-2 friction machine) is used [5-7] in order to physically simulate the formation of a lubricating film in a heavily loaded friction pair. To study the change in the thickness of an oil film formed in the "roller-roller" friction pair we useaweight-of-coating test. In particular,it is an analytical balance with a measurement accuracy of $0.0001 \mathrm{~g}$. The general view of the laboratory setup and the installed rollers are shown in Figure 2.

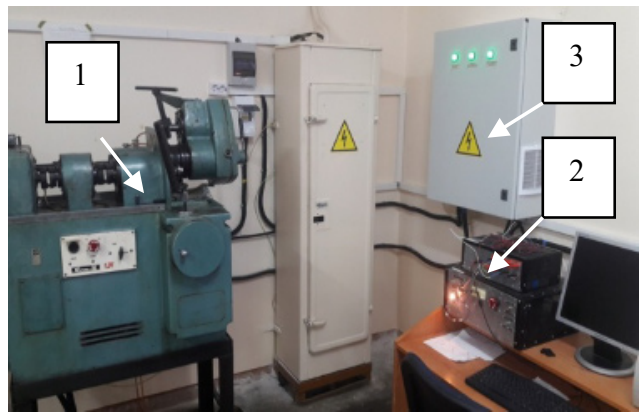

a)

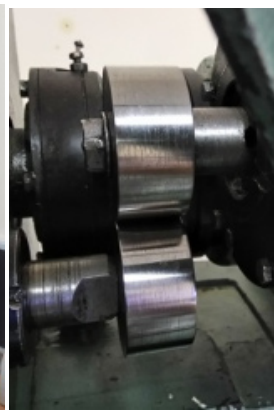

b)

Fig. 2. a) General view of the laboratory setup; b) the "roller-roller" friction pair

The friction machine (Fig. 2a) consists of actual mechanical and measuring units (item 1), a unit for processing and transforming information (item 2) and a switchboard cubicle (item 3) [7].

To carry out the experiments, three pairs of rollers with different microtopography of surfaces are made. The variants of the microtopography of surfaces are obtained by turning, grinding and polishing.

In the course of the experiments, the clamping force and the rotary velocity are changed, thereby providing a specific load per unit contact length of 15,60 , and $100 \mathrm{kN} / \mathrm{m}$ and a rolling speed of $0.5,1.3$ and $2.0 \mathrm{~m} / \mathrm{s}$ at the contact. At the same time, a lubricant with a viscosity class of 220 and a mass of $0.03 \mathrm{~g}$ is fed to the upper roller. For each pair of rollers, five experiments are carried out at the corresponding loads and rolling speeds. In total, 135 tests and measurements are carried out.

The square of a lubricating film emergin gat the contact is measured with the help of samples taken from the surfaces of the rollers by placing a grid with a cell size of $1 \mathrm{~mm}$ in Photoshop CS6 program.A lubricating film thickness $h_{0}$ is defined as a ratio of mass of a supplied lubricant $\mathrm{m}$ sm to the product of its density $\rho$ and square of a lubricating film $S_{c n}$, i.e. $h_{0}=m_{C M} /\left(\rho S_{c n}\right)$. Taking into account the precision of measurements, the calculation accuracy is no less than $85 \%$. Table 1 shows average values $h_{0}$ obtained by experiments. 
The values of the relative oil absorption capacityare set according to the results obtained in [8-10].

\section{Research results and discussion}

The values of lubricating film thicknesses, calculated on the basis ofthe mathematical relationships $[11,12]$, turn out to be significantly lower than those obtained in the course of the experimental studies. This discrepancy is due to the fact that known equations do not take into account a rough layer characterized by certain oil absorptioncapacity, though the latter makes an additional contribution to the formation of a lubricating film.

Some values of lubricating film thicknesses, obtained by generalizing theoretical data on dependence10 and as a result of ourexperimental studies, are shown in Fig. 3.
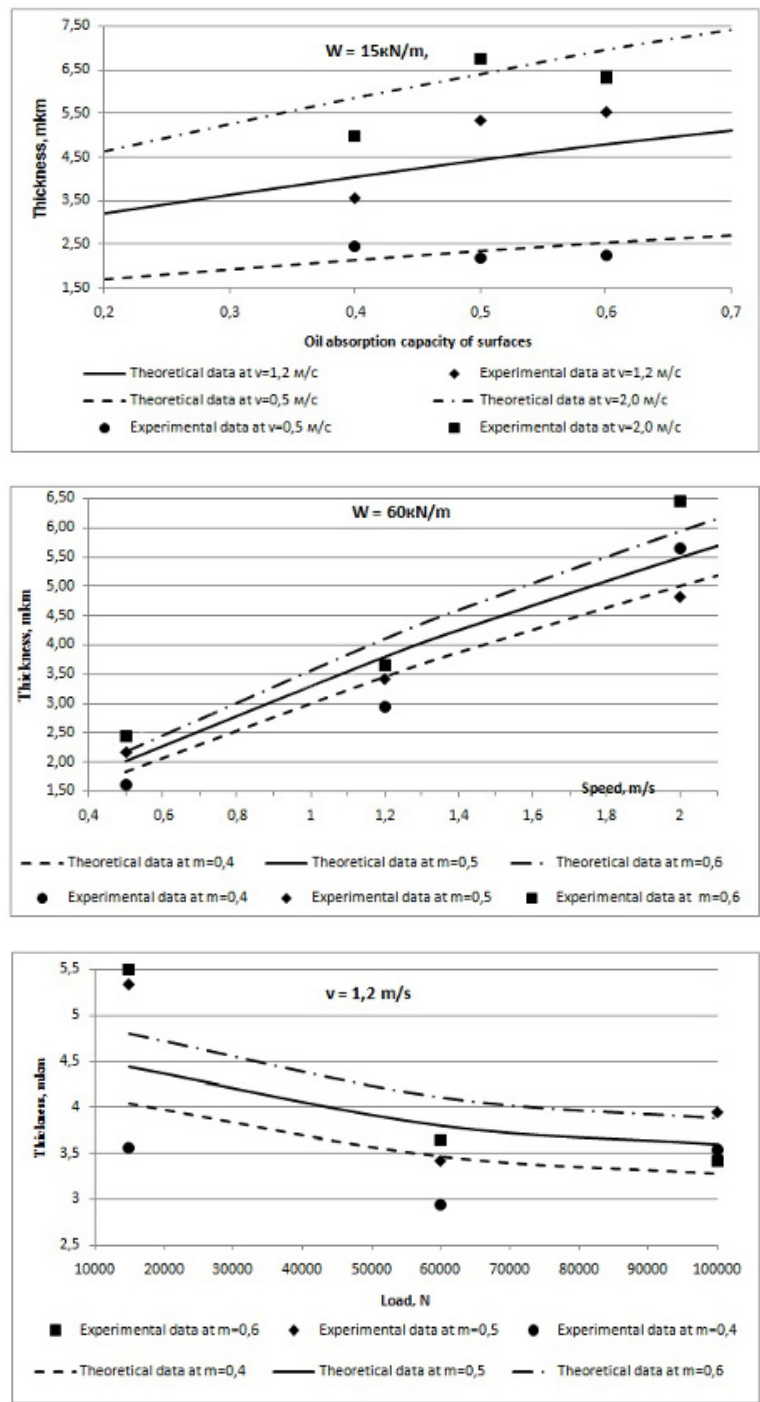

Fig. 3. The results of theoretical and experimental studies of thicknesses of a lubricating film 
The analysis of the presented diagrams shows that with an increase ofa load force acting on contact surfaces in friction assemblies, there appears a decrease in a lubricating filmthickness. The intensity of the changes is more noticeablein the region of low values of loads than in the region of the increased ones. In the range of changes from 15 to $100 \mathrm{kN}$ $/ \mathrm{m}$, i.e.6.7 times, the lubricating film thickness decreases 1.23 times.

Table 1. Thicknesses of a lubricating film, microns, in a contact zone of a friction pair "roller-roller" under various conditions of: oil absorption capacityof surfaces, specific loads and rolling speeds

\begin{tabular}{|c|c|c|c|c|c|c|c|c|}
\hline \multicolumn{3}{|c|}{ Grinding $(\mathrm{m}=0,6)$} & \multicolumn{3}{|c|}{ Turning $(\mathrm{m}=0,5)$} & \multicolumn{3}{|c|}{ Polishing $(\mathrm{m}=0,4)$} \\
\hline \multicolumn{9}{|c|}{ Rolling speed, $\mathrm{m} / \mathrm{s}$} \\
\hline 0,5 & 1,2 & 2,0 & 0,5 & 1,2 & 2,0 & 0,5 & 1,2 & 2,0 \\
\hline \multicolumn{9}{|c|}{ Specific load $15 \mathrm{kN} / \mathrm{m}$} \\
\hline $\begin{array}{l}2,24 \\
(2,54)\end{array}$ & \begin{tabular}{|l|}
5,52 \\
$(4,80)$
\end{tabular} & \begin{tabular}{|l|}
6,32 \\
$(6,95)$
\end{tabular} & \begin{tabular}{|l}
2,19 \\
$(2,35)$
\end{tabular} & \begin{tabular}{|l|}
5,33 \\
$(4,44)$
\end{tabular} & $\begin{array}{l}6,75 \\
(6,43)\end{array}$ & \begin{tabular}{|l}
2,46 \\
$(2,14)$
\end{tabular} & $\begin{array}{l}3,56 \\
(4,04)\end{array}$ & \begin{tabular}{|l}
4,98 \\
$(5,86)$
\end{tabular} \\
\hline \multicolumn{9}{|c|}{ Specific load60kN $/ \mathrm{m}$} \\
\hline \begin{tabular}{|l|}
2,44 \\
$(2,18)$
\end{tabular} & \begin{tabular}{|l|}
3,65 \\
$(4,11)$ \\
\end{tabular} & \begin{tabular}{|l}
6,45 \\
$(5,96)$ \\
\end{tabular} & $\begin{array}{l}2,17 \\
(2,01)\end{array}$ & \begin{tabular}{|l|}
3,42 \\
$(3,80)$ \\
\end{tabular} & $\begin{array}{l}4,82 \\
(5,51) \\
\end{array}$ & \begin{tabular}{|l|}
1,62 \\
$(1,84)$ \\
\end{tabular} & \begin{tabular}{|l}
2,95 \\
$(3,47)$ \\
\end{tabular} & \begin{tabular}{|l|}
5,63 \\
$(5,03)$ \\
\end{tabular} \\
\hline \multicolumn{9}{|c|}{ Specific load $100 \mathrm{kN} / \mathrm{m}$} \\
\hline $\begin{array}{l}1,83 \\
(2,06) \\
\end{array}$ & $\begin{array}{l}3,42 \\
(3,89)\end{array}$ & \begin{tabular}{|l}
6,76 \\
$(5,63)$ \\
\end{tabular} & $\begin{array}{l}1,67 \\
(1,90)\end{array}$ & \begin{tabular}{|l|}
3,95 \\
$(3,59)$ \\
\end{tabular} & $\begin{array}{l}4,90 \\
(5,21)\end{array}$ & \begin{tabular}{|l|}
1,40 \\
$(1,73)$
\end{tabular} & \begin{tabular}{|l}
3,54 \\
$(3,28)$ \\
\end{tabular} & \begin{tabular}{|l|}
4,23 \\
$(4,75)$ \\
\end{tabular} \\
\hline
\end{tabular}

Note Values in parentheses are calculated on the dependence (10).

An increase in a lubricating film thicknessis facilitated by a rate of relative displacement of contacting surfaces and oil absorption capacityof their rough layers. An increase in speed from 0.5 to $2.0 \mathrm{~m} / \mathrm{s}$, i.e. 4 times, leads to an increase in a lubricating film thicknessby an average of 2.8 times. A change in the relative oil absorption capacityfrom 0.4 to 0.6 increases a lubricating film thicknessby about 1.6 times.

Thus, the parameter of relative oil absorptioncapacity of rough contacting surfaces has the greatest influence on the change in a lubricating film thicknessin friction assemblies.

According to GOST 18855-94 (ISO 281-89), acorrected rating life of special bearing properties and special operating conditions are calculated by the formula:

$$
L_{\mathrm{na}}=a_{1} a_{2} a_{3} L_{10}
$$

where $\mathrm{L}_{10}$, standard rating life;

$\mathrm{a}_{1}$, coefficient, correcting life according to reliability;

$a_{2}$, coefficient correcting life depending on special properties of a bearing;

$\mathrm{a}_{3}$, coefficient that corrects life depending on operating conditions of a bearing.

The value of $a_{1}$ coefficient is established and regulated by GOST, $a_{2}$ coefficient is determined by a manufacturer of bearings, and, in general, does not exceed 1.0. According to GOST, $a_{3}$ coefficient can be more than 1 under favorable lubrication conditions.

The lubrication conditions and the mode of friction between contacting surfaces is determined by the parameter $\lambda$, which depends on the thickness of a lubricating layer. In this regard, coefficient $a_{3}$ is proposed to be determined as follows:

$$
\begin{aligned}
& \text { at } 3 \leq \lambda \geq 7, a_{3}=1 ; \\
& \text { at } 3<\lambda, a_{3}=0,8 ; \\
& \text { at } \lambda>7, a_{3}=1,1 .
\end{aligned}
$$

This approach to determine the life is also valid for gears used in metallurgical units. 


\section{Results}

1. Using main theses of the contact-hydrodynamic theory of lubrication of heavy-duty tribo-couplings, an equation is obtained, which is supplemented by a complex oil absorption parameter. The equation takes into account geometric (radius of curvature of contacting bodies)and physical (speed of moving surfaces) characteristics of contacting surfaces, as well asaprocessingmethod. The dependence makes it possible to calculate a thickness of a lubricating layer when surfaces are in contact,regarding their relative displacement and oil absorptioncapacity of theirrough layers.

2. The experimental studies carried out in laboratory conditions with an aim to determine a thickness of a lubricating layer between contacting surfaces shows that oil absorption capacityof rough layers has the greatest influence on the change in the thickness of a lubricating film in friction assemblies.

It is determined that with an increase in a load force acting on the contact of surfaces in friction assemblies, thethickness of a lubricating film decreases. In the region of low values of a load force, the intensity of changes is more noticeable than in the region of the increased ones.In the range of changes from 15 to $100 \mathrm{kN} / \mathrm{m}$, i.e. 6.7 times, the thickness of a lubricating film decreases 1.23 times. An increase in speed from 0.5 to $2.0 \mathrm{~m} / \mathrm{s}$, i.e. 4 times, leads to an increase in the thickness of a lubricating film by an average of 2.8 times. The change in the relative oil absorption capacityfrom 0.4 to 0.6 increases the thickness of a lubricating film by about 1.6 times. The convergence of the experimental values and those calculated by the proposed method was $85 \%$.

3. A coefficient is proposed which corrects the basic resource of rolling bearings and gear drives. It can take the values from 0.8 to 1.1 , depending on the oil absorption capacity of contacting rough layers and the operating parameters of machine and assembly units.

\section{Discussion}

The comparison of the obtained data on the thickness of a lubricating layer (regarding the oil absorption capacity which was experimentally obtained and analytically calculated) shows that the thickness of a lubricating layer is higher than it was previously thought. Analyzing the data obtained, it is found that the parameter of relative oil absorption capacityof rough contacting surfaces has the greatest influence on the change in the thickness of a lubricating film in friction assemblies.

The verification of the validity of the developed analytical model for calculating the thickness of a lubricating layer in a heavy-duty friction pair is carried out on modern laboratory equipment according to an original technique, taking into account various parameters of interaction of contacting surfaces in the presence of a lubricant. It is noted that an increase in the thickness of the lubricating film is facilitated by the rate of relative displacement of contacting surfaces and the oil absorption capacityof their rough layers. With an increase in the load acting on the contact surfaces in friction assemblies, the thickness of a lubricating film decreases.

This work is carried out within a framework of the government order (No. FZRU-2020-0011) of the Ministry of Science and Higher Education of the Russian Federation

\section{References}

1. Kovalevsky, V.F. Tribotechnical characteristics of sliding friction pairs with a sandretaining relief formed by drip-adhesive technology / V.F. Kovalevsky // Omsk Scientific Bulletin. 2013. No. 2. S. 78-81. 
2. Pashovkin, S.A. Model of contact of rough surfaces taking into account oil absorption in the contact zone // Izvestiyavuzov. Mechanical engineering. 2008. \# 12. S. 67-72.

3. Shelkova, A.N. Criteria for the formation of structures and parameters of processing systems that ensure the specified operational properties of hardened large-modular gear wheels. Shelkova, E.V. Mironenko, A.A. Klochko // Modern technologies in mechanical engineering. 2013, type. 8.S. 185-200.

4. Kuznetsov, V.P. Modeling and research of the formation of flat-topped microrelief of friction surfaces with lubricating micropockets during multi-purpose processing of parts / V.P. Kuznetsov, O.V. Dmitrieva // Bulletin of the Tomsk Polytechnic University. 2011. T 319. No. 2. S. 35-40.

5. Terentyev, D.V. Influence of operating modes and oil absorption of contact surfaces on the thickness of the lubricating film in heavily loaded friction units of metallurgical units / D.V. Terentyev, N.N. Ogarkov, S.I. Platov, A.V. Kozlov // Ferrous metals. 2018. No. 9. S. 60-64.

6. Elastohydrodynamic friction mode as a method of surface finishing excluding burnishing, Materials ScienceForum, 2019, vol. 946 MSF, p. 732-738, Kharchenko, M.V. and Kononov, V.N. and Zambrgitckaya, E.S. DOI: 10.4028 / www.scientific.net / MSF.946.732

7. Study of the conditions for the formation of an adsorption lubrication mode of heavily loaded friction couples with modeling in a laboratory setup // Journal of Friction and Wear, 2019,40,4, p. 277-283, Levantsevich, M.A. and Kharchenko, M. V. and Dema, R. R. DOI: 10.3103 / S106836661904007X

8. Ogarkov, N.N. Oil absorption capacity of the contact surfaces in metal-forming processes / N.N. Ogarkov, S.I. Platov, E.S. Shemetova, D.V. Terentev, V.A. Nekit, M.N. Samodurova // Metallurgist. 2017. T. 61. No. 1-2. S. 58-62.

9. Ogarkov, N.N. Oil absorption of contact surfaces in metal pressure processing / N.N. Ogarkov, S.I. Platov, E.S. Shemetova, M.N. Samodurova, D.V. Terentyev, V.A. Nekit // Metallurg. 2017. No. 1. S. 79-82.

10. Search for effective lubricants and lubricating coatings for stamping rod fasteners from stainless steels OS Zhelezkov, AN Abramov, T.Sh. Galiakhmetov. // Bulletin of the Magnitogorsk State Technical University. G.I. Nosov. 2017.Vol. 15.No. 4.P. 4754.

11. Kodnir, D.S. Elastohydrodynamic calculation of machine parts / D.S. Kodnir, E.P. Zhilnikov, Yu.I. Bayborodov. M .: Mashinostroenie, 1988.160 p.

12. Kodnir, D.S. Contact hydrodynamics of lubrication of machine parts / D.S. Codnir. Moscow: Mashinostroenie, 1976.304 p. 